\title{
Displasia ectodérmica hipohidrótica: Reporte de casos.
}

\author{
Hypohydrotic ectodermal dysplasia: Report of case.
}

\author{
Eda Mariola Dolmo Velásquez*, Misael Alonso Pineda**, Hector Rubén Caballero Castro***
}

\section{RESUMEN}

La Displasia Ectodérmica Hipohidrótica (DEH) es una genodermatosis que se caracteriza por presentar alteraciones en las estructuras derivadas del ectodermo, frecuentemente se da la triada: hipohidrosis, hipotricosis e hipodoncia. El síndrome puede manifestarse como herencia autosómica dominante o recesiva y también como herencia ligada al sexo, la forma más frecuente es la de herencia recesiva relacionada al cromosoma $X$ con sujetos de sexo masculino afectados y de sexo femenino portadores. Puede ocurrir a través de mutaciones autosómicas, de las cuales las del gen EDA1 son responsables del $58 \%$ de los casos. La DEH presenta tasa de mortalidad infantil entre $2 \%$ y $20 \%$, dependiendo de la precocidad del diagnóstico y de los protocolos de tratamiento. Este artículo presenta un paciente de 23 meses de edad quien había sido hospitalizado por otrapatología y se refirió al Instituto Hondureño de Seguridad Social (IHSS), por observar cabello hipopigmentado, escaso, fino, ausencia de pestañas y cejas, dientes cónicos e hipohidrosis: por lo que se diagnostica displasia ectodérmica hipohidrótica, quedando pendiente la realización de biopsia de piel y exámenes genéticos debido a que no se cuenta con el equipo médico necesario. Por tal motivo, no se conoció el patrón de segregación.

\section{PALABRAS CLAVE}

Displasia Ectodérmica Hipohidrótica Autosómica Recesiva, Anodoncia, Hipotricosis.

\footnotetext{
* Médico residente de primer año de Pediatría. Escuela Universitaria de las Ciencias de la Salud. Universidad Nacional Autónoma de Honduras, Valle de Sula. EUCS, UNAH-VS.

**Médico residente de tercer año de Pediatría. EUCS, UNAH-VS.

***Especialista en dermatología Pediátrica. Instituto Hondureño de Seguridad Social Regional Norte.

Dirigir correspondencia a: edmadove@yahoo.es

Recibido: 07 de noviembre 2016 Aprobado: 08 de agosto 2017
}

\begin{abstract}
Hypohidrotic Ectodermal Dysplasia (DEH) is a genodermatosis that is characterized by alterations in the structures derived from the ectoderm. Frequently occurs this triad: hipohidrosis, hypotrichosis and hypodontia. The syndrome can manifest as autosomal dominant or recessive inheritance and also as sex-linked inheritance, the most common form is recessive $X$ chromosome linked inheritance, subjects affected are males and femalesare only carriers. It can occur through autosomal mutations, and in these, the gene EDA 1 are responsible for $58 \%$ of cases. It presents infant mortality rate between $2 \%$ and $20 \%$, depending on the early diagnosis and treatment protocols. This article presents a 23-month-old patient who had been hospitalized for another condition, however observing the above mentioned features in the physical examination, he was studied in more detail in the IHSS.
\end{abstract}

\section{KEY WORDS}

Hypohidrotic Ectodermal Dysplasia Autosomal Recessive, Anodontia, Hypotrichosis.

\section{INTRODUCCIÓN}

El ectodermo es uno de los componentes embrionarios primordiales. En torno a la tercera semana de desarrollo experimenta una subdivisión que da lugar al neuroectodermo, que origina el sistema nervioso y al ectodermo mismo, que recubrirá toda la superficie embrionaria y formará la epidermis, sus anexos y el esmalte dental. Por tanto, del ectodermo derivan no solo el pelo, los dientes, las uñas y las glándulas sudoríparas; sino también el sistema nervioso central, el sistema nervioso periférico, el ojo, el oído y la nariz, así como las glándulas ecrinas, mamarias y pituitaria. ${ }^{(1-3)}$ 
Las primeras descripciones de casos clínicos que podrían corresponderse con lo que ahora conocemos como DEH se remontan a 1792. En 1848 Thurman definió la displasia ectodérmica anhidrótica (DEH) como una entidad independiente. Posteriormente se describieron casos similares como el presentado por Wedderhorn y publicado en1875 por el naturalista Charles Darwin. El término fue definido finalmente por Weech en 1929. ${ }^{(1)}$

Actualmente se reconocen tres patrones de segregación de la Displasia ectodérmica hipohidrótica (DEH): Uno recesivo ligado al cromosoma $\mathrm{X}$ que además incluye una variante con compromiso inmunológico denominada DEHID, y las variantes autosómicas recesivas y dominante. Es de resaltar que variantes autosómicas de la DEH son indistinguibles clínicamente de las formas ligadas al cromosoma $X$ (con excepción de la DEH-ID por la inmunodeficiencia), probablemente porque en todas está alterada una vía única de señalización, por lo cual el diagnóstico diferencial de estos individuos, afectados y portadores, suele realizarse mediante análisis genético con pruebas específicas. $^{(2,3)}$

El 95\% de los pacientes con DEH seleccionados al azar presentan la forma ligada a $\mathrm{X}$ y el $5 \%$ restante la forma autosómica dominante (AD) o recesiva (AR). A veces la historia familiar puede aclarar el modo de herencia y si los casos son únicos en la familia sólo la identificación del defecto genético definirá el origen de la enfermedad. ${ }^{(3)}$

De modo general, se diferencian 2 grupos de trastornos: uno caracterizado por la aplasia o hipoplasia de los derivados ectodérmicos, que fracasan en su desarrollo y diferenciación por la ausencia de señales recíprocas específicas entre ectodermo y mesénquima y otro en el que la característica más llamativa es la queratodermia palmoplantar, que se presenta en asociación con otras manifestaciones cuando se afectan otros epitelios altamente especializados. En las últimas décadas se ha logrado identificar el gen responsable en al menos 30 entidades, permitiéndonos entender los mecanismos patogénicos y su correlación con la clínica. ${ }^{(4)}$ Se estima una frecuencia de 1 por cada 10.000 a 1 por cada 100.000 nacidos vivos. $^{(5)}$

El objetivo de esta publicación, es dar a conocer las características clínicas de la DEH, para realizar diagnósticos más temprano.

\section{CASO CLINICO}

Se trata de paciente masculino de 23 meses de edad que acudió al Hospital Nacional Dr. Mario Catarino Rivas con historia de tos no productiva de 4 días de evolución, acompañado de rinorrea hialina, fiebre no cuantificada pero subjetivamente alta y dificultad respiratoria por lo que fue hospitalizado para tratar patología respiratoria durante dos días con mejoría de dicho cuadro clínico. Siendo evidente fenotipo característico (pelo de cuero cabelludo plateado, escaso y fino, vello corporal también escaso y dientes caninos pequeños y de forma cónica). Fue referido al Instituto Hondureño de Seguridad Social para continuar estudios histopatológicos e inmunológicos, sospechando en ese momento Síndrome de Griselli debido al hallazgo de cabellos plateados y además del diagnóstico de desnutrición proteíco calórica aguda con déficit del $22 \%$.

Con los siguientes antecedentes: madre de19 años de edad, parto sin complicaciones, hospitalizado al nacimiento en sala de neonatología durante 5 días por sepsis neonatal temprana. Con leve retraso en el desarrollo del lenguaje. Antecedentes familiares patológicos; tío y primo materno que tienen el mismo fenotipo pero que nunca han sido diagnosticados.

En la exploración física: Peso: 8.5 kg, talla: 83 
$\mathrm{cm}$, perímetro cefálico: $44.5 \mathrm{~cm}$. Sin alteraciones conductuales pero con leve retraso en el desarrollo del lenguaje, cabello hipopigmentado, escaso, fino, ausencia de pestañas y cejas. (Ver figura No.1) Dientes en forma de cono y pequeños, piel brillante, seca y tirante (apergaminada) con hipohidrosis. (Ver figura No. 2)

Figura No. 1: Cabello del paciente.

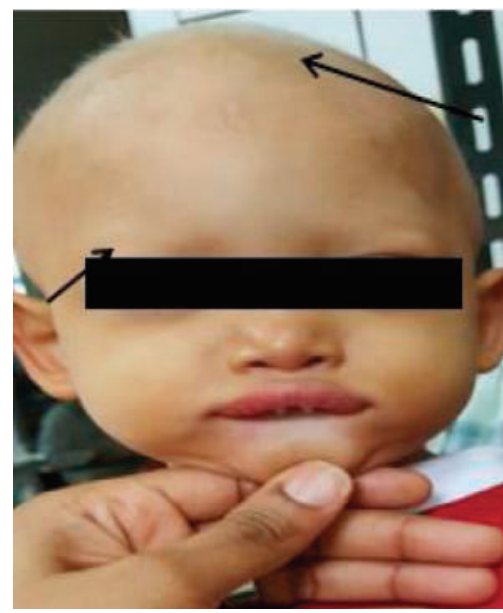

La flecha superior señala cabello hipopigmentado, escaso y fino. La flecha inferior muestra la ausencia de cejas.

Figura No 2. Dientes del paciente.

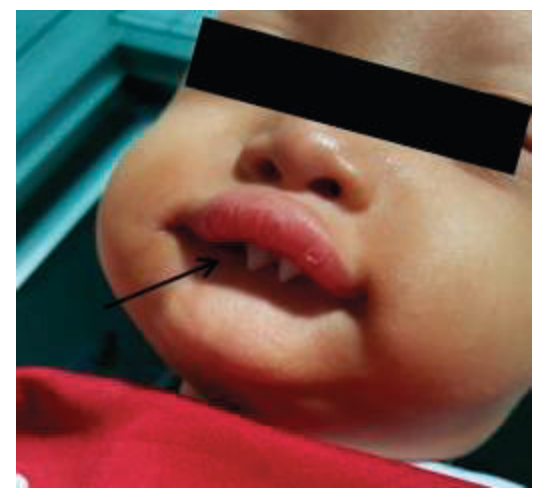

La Flecha indica hipodoncia, dientes en forma cónica.

Los exámenes laboratoriales mostraron: Calcio: 10.1mmo/l, Sodio: 138mmo/l (135 mmo/l -145 $\mathrm{mmo} / \mathrm{l})$, potasio: $5.25 \mathrm{mmo} / \mathrm{l}$. $(3.5 \mathrm{mmo} / \mathrm{l}-5.5$ $\mathrm{mmo} / \mathrm{l})$ lgA: 78, (22-159), lgE: 116, C3:113. $\mathrm{mg} / \mathrm{dl}$, biometría hemática: hemoglobina 10.4 gr/dl (11.5 gr/dl -12.5 gr/dl), plaquetas: 538 x103/uL (150 x 103/uL -350x 103/uLglóbulos blancos 17.0 x 103/uL (5103/uL-15.5103/uL).
Exámenes de imagen mostraron ultrasonido abdominal y tomografía axial computarizada cerebral sin alteración. Quedando pendiente la realización de biopsia de piel y exámenes genéticos debido a que no se cuenta con el insumo médico necesario. Por tal motivo no se conoció el patrón de segregación, sin embargo, los antecedentes familiares afianzaban el diagnóstico.

\section{DISCUSION}

La Displasia Ectodérmica (DE) es un síndrome constituido por un grupo heterogéneo de enfermedades que resultan como consecuencia de defectos variables en dos o más tejidos derivados del ectodermo embrionario, incluyendo la piel, glándulas sudoríparas, cabello, uñas y dientes. Igualmente se han reportado anomalías en otros órganos y sistemas. ${ }^{(1)} \mathrm{Se}$ estima una frecuencia de 1 por cada 10.000 a 1 por cada 100.000 nacidos vivos. ${ }^{(5)}$ Presenta tasa de mortalidad infantil entre $2 \%$ y $20 \%$, dependiendo de la precocidad del diagnóstico y de los protocolos de tratamiento. ${ }^{(6)}$ Observamos como coinciden la diferentes afecciones propias del ectodermo en el paciente presentado en este caso clínico.

El gen responsable de la $D E H$ se sitúa en el locus q12-q13 del cromosoma $X$, constando de 12 axones, siendo ocho de ellos encargados de codificar una proteína transmembrana (EDA-A) involucrada en el desarrollo ectodérmico, cuyas mutaciones originan la patología que caracteriza dicha enfermedad. (7) Debido a la falta de insumos en los dos hospitales donde se ha brindado tratamiento al paciente, no se lograron realizar pruebas genéticas para identificar el locus del gen responsable.

La DEH altera la apariencia física normal de los pacientes que la padecen, entre las anomalías que se presentan en todas las formas de segregación de la $\mathrm{DEH}$, existen tres características cardinales: anomalías dentales usualmente 
hipodoncias, además hipohidrosis e hipotricosis. ${ }^{(1-4)}$ Estas tres anormalidades son evidentes en el paciente de este caso. Segurado et al. describieron los signos confirmatorios de DEH que se enuncian a continuación:

\section{A. Alteraciones del cabello: Hipotricosis en cuero} cabelludo, cejas, pestañas, región púbica y axilar; el cabello es de color claro, corto y quebradizo con posible pérdida o alteración estructural del tallo del pelo que causa una deformación denominada tricorrexis fisurada.

\section{B. Anomalías dentales de número, forma y} tamaño: Pueden presentarse tanto en la dentición primaria como permanente. Entre las manifestaciones dentarias más comunes se encuentran los dientes cónicos o en clavija y la microdoncia pasando por la hipodoncia u oligodoncia, hasta casos más severos con anodoncia, lo cual desencadena un desarrollo deficiente del hueso alveolar, dando apariencia facial de un paciente adulto edentado.

C. Hipohidrosis/anhidrosis: Compromiso mayor de las glándulas de tipo ecrino, seguidas por las apócrinas y las sebáceas, lo cual genera hiperpirexia con una marcada intolerancia al calor, y convulsiones esporádicas. Otras consecuencias de la disminución o ausencia de glándulas son la laringitis crónica, las infecciones pulmonares recurrentes, xeroftalmía, piel seca y dificultad para la masticación y la deglución por disminución de la cantidad de saliva fluida. ${ }^{(1,8)}$

Sumado a las características cardinales, los pacientes con DEH pueden presentar rasgos faciales dismórficos típicos como: frente abultada, orejas puntiagudas de baja implantación, nariz en silla de montar, labios gruesos y evertidos, $^{(1,9)}$ por lo tanto para estos pacientes además de las complicaciones físicas que conlleva este síndrome existirá un gran stress emocional incluso en muchos casos puede sufrir de acoso escolar por ser diferentes físicamente. ${ }^{(9)}$
El diagnóstico diferencial de las displasias ectodérmicas se debe realizar con patologías como: la aplasia de cutis congénita, la disqueratosis congénita, la fiebre de origen desconocido o síndromes progeroides, como el síndrome de Werner o el de Rothmund-Thompson así como con el síndrome de Griselli, todos ellos descartados en nuestro paciente. ${ }^{(10)}$ Todos los anteriores fueron desestimados debido a que nuestro paciente no tenía las características clínicas necesarias para ubicarlo dentro de dichas patologías ya que estas incluían ausencia de piel, hiperpigmentación cutánea, cataratas, alteraciones óseas entre otras, además ninguna de ellas desarrolla hipodoncia o dientes con forma cónica.

El diagnóstico se basa fundamentalmente en la sintomatología y en el estudio genético, siendo la clave la disminución o falta de sudoración cuando la temperatura corporal se eleva. También se puede realizar el diagnóstico prenatal de forma invasiva (biopsia de piel fetal o estudio genético de vellosidades coriónicas) o no invasiva, mediante ultrasonografía tridimensional identificando las características faciales a partir de la semana 30 de gestación. ${ }^{(7)}$ En Honduras es casi imposible por no decir completamente imposible poder realizar estudios genéticos por lo que el diagnóstico es basado en la clínica, además el mayor porcentaje de madres no tienen control prenatal básico adecuado lo que dificulta la posibilidad de realizar una detección de caso precozmente y los métodos diagnósticos necesarios en estos casos están fuera del alcance económico de la mayoría de la población.

Durante las primeras semanas de vida es complicado reconocer la presencia de DEH en el recién nacido y es importante pensar en ella en los casos de aparición de fiebre de origen desconocido o hiperpirexia como diagnóstico diferencial frente a la sepsis neonatal, con los beneficios que esto conlleva en cuanto a la 
realización de medidas diagnósticas y terapéuticas. ${ }^{(7)}$ Si se realiza diagnóstico al nacimiento se deben usar factor de crecimiento epidérmico, por lo menos en los primeros 2 meses de vida como medida de activación y desarrollo de las glándulas sudoríparas. ${ }^{(8)}$ Nuestro paciente fue hospitalizado a su nacimiento por sepsis neonatal durante 5 días, sin embargo no contamos con el expediente previo sin embargo nos hace pensar que tan acertado fue el diagnóstico en ese momento, o si lo que presento fue un cuadro de hiperpirexia pasada por alto.

En general los exámenes de laboratorio no son necesarios para hacer el diagnóstico. Ante la sospecha clínica del síndrome, se sugiere realizar una biopsia de piel (zona hipotenar de la mano) para documentar la reducción o total ausencia de glándulas ecrinas. Una prueba fehaciente y muy útil para demostrar la ausencia de sudoración es la prueba con nitrato de pilocarpina por vía subcutánea para estimular la sudoración. Otra alternativa para demostrar la anhidrosis es aplicar lugol y almidón en la región palmar. La prueba es positiva si el lugol, comúnmente amarillento, cambia su tonalidad a violácea. Es negativa si no se presenta ningún cambio. Las cuales no son necesarias ante los hallazgos en la biopsia de piel. ${ }^{(9)}$ En el paciente de este caso, no fue necesario realizar ninguna de estas pruebas porque era evidente la anhidrosis y los rasgos físicos.

El tratamiento reconocido en la literatura es exclusivamente sintomático y preventivo e incluye evitar ejercicios físicos, mejorar las condiciones de vida del paciente como tratar de mantenerse en climas fresco, con protección visual, medidas sintomáticas que mantengan la hidratación de piel y mucosas así como apoyar los cuidados de la piel con cremas hidratantes y protectores solares. A nivel odontológico el manejo tiene propósito rehabilitador y funcional de las estructuras dentarias con el uso de prótesis o implantes y la utilización de resinas para la rehabilitación de dientes con alteraciones en su estructura. El tratamiento debe ser individualizado dependiendo de la gravedad del caso, de acuerdo con la edad y desarrollo dental del individuo. ${ }^{(8,9)}$

La DEH es una entidad en la cual el pronóstico de sobrevida es muy bueno. Si la entidad se reconoce desde temprana edad y es tratada adecuadamente mediante el apoyo con un equipo multidisciplinario de salud, la esperanza de vida no debería de verse afectada y la calidad de vida del paciente podría mejorar de manera exitosa. ${ }^{(6-9)}$

En conclusión; a pesar de la baja incidencia de $\mathrm{DEH}$, consideramos necesario que esta enfermedad pueda ser reconocida y sospechada por pediatras y neonatólogos, ya que su manejo debe ser multidisciplinario involucrando profesionales como pediatras, dermatólogos, genetistas, dentistas, ortodoncistas y cirujanos plásticos. El diagnóstico precoz permite evitar el efecto deletéreo que puede acarrear la hipertermia y la susceptibilidad a infecciones respiratorias.

Es recomendable evitar y tratar la hipertermia aguda con control ambiental y baños de agua fría, que contribuirán a evaporar el calor corporal. Se debe informar al paciente y a la familia medidas para evitar el calor, ejercicio físico intenso, climas cálidos además de aconsejar deportes acuáticos. 


\section{BIBLIOGRAFÍA}

1. García-Martín P, Hernández-Martín A, Torrelo A. Displasias ectodérmicas: revisión clínica y molecular. [Internet]. 2013 [citado 2016 Jun 16] ; 104( 6 ):451-470. Disponible en: http://www.actasdermo.org/es/displasias-ectodermicas-revision-clinica-mole cular/articulo/S0001731012004073/.

2. Marín Botero ML, Espinal Botero G, Arroyo Fuentes T, Posso Zapata MV, David Pérez M, Castañeda Peláez DA, et.al. Displasia ectodérmica hipohidrótica: Reporte de casos. Av. Odontoestomatologia [Internet]. 2013 [citado 2016 Jun 16] 29 (1): 11-23.

Disponible en: http://scielo.isciii.es/pdf/o donto/v29n1/original1.pdf.

3. Guillén Navarro E, Ballesta Martínez MJ, López González V. Displasia ectodérmica hipohidrótica. . [Internet]. 2010 [citado 2016 Jun 13] ; 1(1):13-7. Disponible: http:// www.aeped.es/sites/default/files/docu mentos/displasia_ectodermica_hipohidro tica.pdf.

4. Guillén Navarro E, Ballesta Martínez MJ, López González V. Displasia ectodérmica hipohidrótica. . [Internet]. 2010 [citado 2016 Jun 13] ; 1(1):13-7. Disponible: http:// www.aeped.es/sites/default/files/docu mentos/displasia_ectodermica_hipohidro tica.pdf.

5. Loreto Treviño F, Jaime Aviara E, Vagas MJ, Alarcon RC. DisiplinaEtodermicaHipohidroticaAutosomica Dominante. [Internet]. 2012 [citado 2016 Jun 10] ; 1(1):1-3. Disponible: http://www.revistaanacem.cl/ pdf/vol6/n2/displasia.pdf.

6. Alves N, Oliveira R, DeanaNF. Displasia ectodérmica hipohidrótica - un síndrome de interés para la odontología. Int. J. Odontos- tomat. [Internet]. 2012 [citado 2016 Jun 18]; 6(1):45-50. Disponible en: http://www. scielo.cl/pdf/ijodontos/v6n1/art06.pdf.

7. Miranda Díaz Belkis Tamara, Rodríguez Casas MíriamMarlen. Displasia ectodérmica hipohidrótica: Presentación de un caso. Rev. Med. Electrón. [Internet]. 2009 Jun [citado 2016 Jun 18] ; 31( 3 ): . Disponible en: http://scielo.sld.cu/scielo.php?script= sci_arttext\&pid=S1684-18242009000300 014\&lng=e.

8. Marín Botero ML, Espinal Botero G, Arroyo Fuentes TM, Posso Zapata MV, David Pérez M, Castañeda Peláez DA, et al. Displasia ectodérmica hipohidrótica: Reporte de casos. AvOdontoestomatol [Internet]. 2013 Feb [citado 2016 Jun 19]; 29(1): 11-23. Disponible en: http://scielo.isciii.es/scielo. php?script=sci_arttext\&pid=S0213-1285 2013000100002\&lng=es.

9. Díaz de la Vega ST, CardemilBalari A, Cardemil Van Der Schraft V, Cárdenas Illanes MG. Manejo Multidiciplinario y Preventivo de Displasia Ectodérmica Hipohidrótica: A Propósito de 3 Casos en una Familia. [Internet]. 2001 [citado 2016 Jun 10] ; 1(1): 1-2.Disponible:http://www.radlalima2015. org/posters/TOSO\%20Stefania\%20(CHI) $\% 20$ manejo\%20multidiciplinario\%20 y\%20preventivo\%20de\%20displasia\%20 ectodermica.pdf.

10. Pipa Vallejo A, López-Arranz Monje E, González García M. Tratamiento odontológico en la displasia ectodérmica: Actualización. AvOdontoestomatol [Internet]. 2006 Jun [citado 2016 Jun 19] ; 22( 3 ): 171-176. Disponible en: http://scielo.isciii.es/scielo. php?script=sci_arttext \&pid=S0213-12852 006000300003\&lng=es. 